\title{
diSNei: A Collaborative Environment for Medical Images Analysis and Visualization*
}

\author{
Carlos Alberola ${ }^{1}$, Rubén Cárdenes ${ }^{2}$, Marcos Martín ${ }^{1}$, Miguel A. Martín ${ }^{1}$, \\ Miguel A. Rodríguez-Florido ${ }^{2}$, and Juan Ruiz-Alzola ${ }^{2,3}$ \\ 1 ETSI Telecomunicación. University of Valladolid, Spain, \\ caralb@tel.uva.es, \\ http://atenea.tel.uva.es \\ 2 Dep Señales y Comunicaciones. University of Las Palmas de Gran Canaria, Spain, \\ jruiz@dsc.ulpgc.es, \\ http://grtv.teleco.ulpgc.es \\ 3 Dep. Radiology. Harvard Medical School and Brigham \& Women's Hospital, USA
}

\begin{abstract}
In this paper we describe our environment diSNei, a graphical tool for collaborative image analysis and visualization of models created out of slices of volume data; this application allows a number of users to simultaneous and coordinatedly analyze medical images, create graphical models, navigate through them and superimpose raw data onto the models. The application is intended to help physicians interpret data in the case that ambiguous situations may appear, by means of collaboration with other colleagues. It is therefore an integrated environment for expertise interchange among physicians and we believe that it is a powerful tool for academic purposes as well. Other outstanding application features are its being multiplatform, and, particularly, the fact that it can run on NT computers, and the support for stereo rendering so as to obtain a deep sensation of inmersion into the models.
\end{abstract}

\section{Introduction}

Information technologies are nowadays naturally incorporated in most of the professional and social activities; in particular, graphical computer applications ease tremendously the complex process of multidimensional data interpretation. Consequently, an important effort has been focused on this concept, in very different areas, such as geology, meteorology, chemistry and, of course, medical imaging. As far as the medical field is concerned, a number of applications have been created and reported, both in Europe[1] 2] and in the US [3] [4] just to mention a few. Specifically, the joint effort of MIT \& and the Surgical Planning Laboratory (SPL) at Harvard Medical School has given rise to a Web-based environment consisting of Java applets running on any popular browser. This package is a framework for integration of images and textual information and

\footnotetext{
* This paper is a joint effort of two Spanish universities coordinated by the European research grant 1FD97-0881. Alphabetical order has been used in the author's order. Correspondence should be addressed to the last author.
} 
it allows users to combine 3D surface models of anatomical structures, their cross-sectional slices, and textual descriptions about the structures.

Other advanced systems have been reported, as 3DSlicer [5] from SPL, ANALYZE [6], MEDx [7] and MNI [8]. All of these present more extensive applications in image-guided medicine and surgical guidance, and they incorporate powerful tools for segmentation, registration and quantitative data analysis.

All these tools, seem to work as isolated environments, in which a single user 'does the job'. However, inclussion of the possibility of collaboration seems very interesting, as it has been reported elsewhere [9] for one-dimensional signals and [10] for echocardiography.

In this paper we present our package called disNe1 which is an integrated environment for collaborative data analysis and visualization. This environment is totally architecture-independent, easily expandable, and though it was initially intended as a fetal-growth monitoring tool, it is transparent to the source of data to be analyzed. Apart from the classical visualization tools, it incorporates a module for symmetric collaborative work, which, to the best of our knowledge, has not been reported in fetal echography. This type of data have an inherent difficulty in interpretation (specially if data have already been acquired and no further views can be incorporated into the data base) and therefore we believe it will be of interest for diagnosis applications in unclear or ambiguous situations (in which several opinions of experts may disambiguate the situation) and academia and training as well.

In what follows, we describe the system architecture, as well as the functionality currently included in our environment. A number of snapshots taken from the application illustrate it ease of use and its graphical capabilities.

\section{The diSNei Data Analysis and Visualization Environment}

Our package incorporates the facilities of other more classical packages (image segmentation, generation of graphical models out of segmented data, inmersive navigation and so forth) and it also incorporates a module for computer supported collaborative work (CSCW).

Due to the fruitfull activity that characterizes this field, a major issue in diSNei is its modularity; new functions can be easily attached to the graphical user interface (GUI) just with a button, leaving the rest of the environment untouched. Portability is also a major concern, due to the great number of platforms currently in use. These two points are easily achieved by means of portable environments and programming languages such a the Visualization Toolkit (VTK) [11] for data processing and visualization and Tcl/Tk 12] for flow control and GUI design and management. VTK is an open shareware code, so new classes

\footnotetext{
${ }^{1}$ The acronym comes from the Spanish Diseño Integrado de Segmentador y Navegador de Estructuras Internas, i.e. integrated design of a segmenter and a visualizer of inner structures.
} 


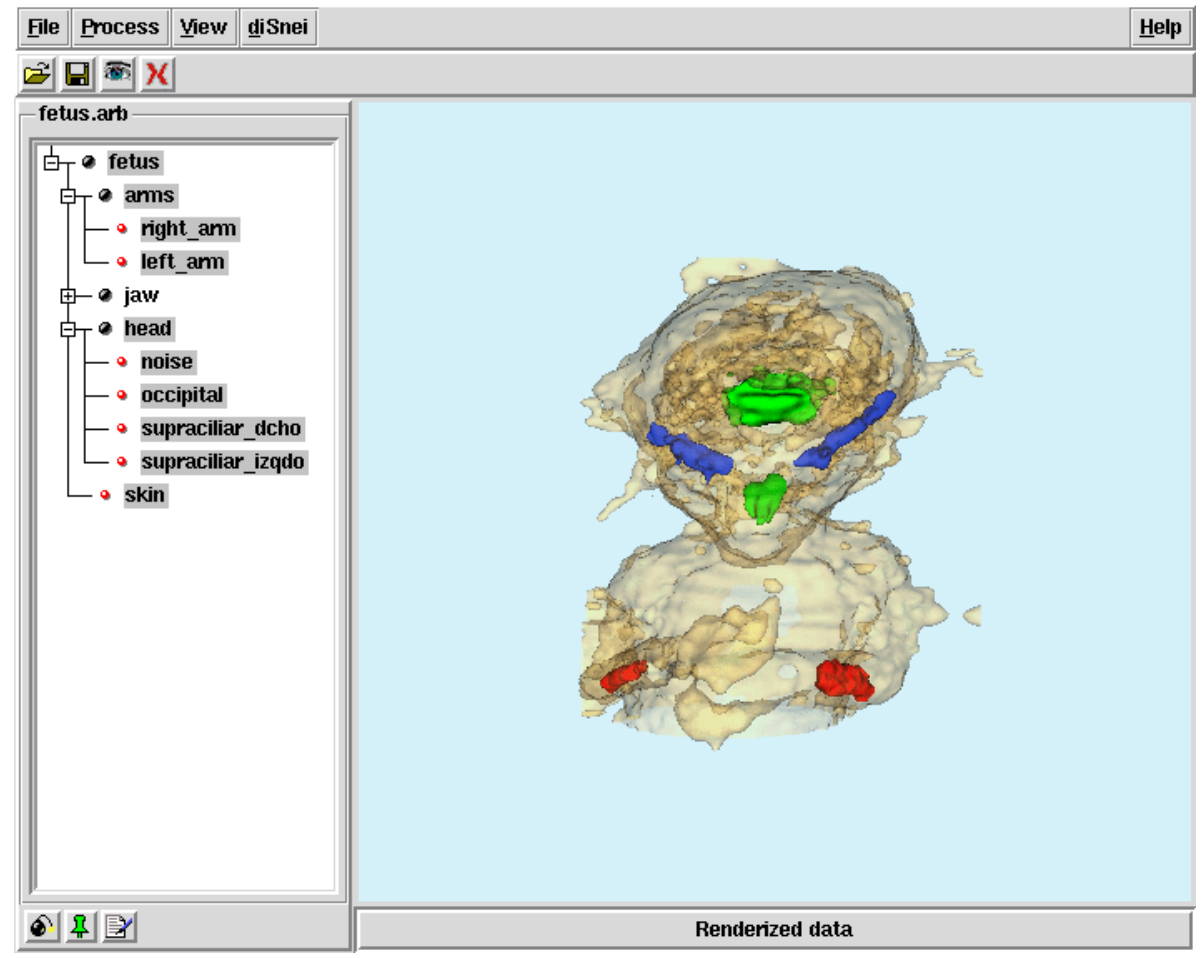

Fig. 1. An example of diSNei for some volume data (specifically, data from a fetus). The GUI shows menu buttons on top, the hierarchically-interpreted data on the left hand side and the navigation window with a semitransparently rendered view.

can be easily incorporated into the environment and made available to the community.

Figure 1 1 shows the diSNei's GUI. As it can be seen it basically consists of four frames, namely, the menu buttons bar, the rendering and navigation window, the hierarchical data structure of organs at disposal, and a status bar. The hierarchical data structure makes it particularly simple to select and highlight organs of interest in the navigation model. Details follow.

\subsection{Image Segmentation with diSNei}

An image segmentation tool is crucial for graphical medical applications since every organ must be properly identified in order to perform any further analysis. Our hypothesis is that data are originally stored as a series of parallel slices; therefore, for the sake of efficiency, our Segmentation Manager Tool (SMTool) starts working in a selected slice with classical segmentation algorithms, but 
then it projects the labels so far obtained onto subsequent slices to benefit from the spatial redundancy inherent in a slice-oriented imaged volume.

As we said in the Introduction, diSNei is clinically-oriented and it is initially aimed at fetal 3D ultrasound image analysis. Therefore we have divided our modules into two categories, namely, clinical and research algorithms. As far as the first category is concerned, our segmentation algorithms are simple since no sophisticated algorithms have proven their success in such an image modality. The segmentation procedure requires human intervention, but we have tried to build a flexible environment to let the operator perform simple operations comfortably. Specifically (see figure 2):

1. The segmentation procedure is governed by the concept of a session. The operator can start a session at anytime, proceed with the segmentation for as long as desired, and then save the segmentation results to disk. If the segmentation procedure is not finished, the operator can retrieve the session in exactly the same stage as it was saved; consequently, fatigue in the operator does not interfere the labelling procedure.

2. The organs and structures are segmented by simple interactions with the mouse: the operator draws a coarse template of the object to extract, and then a region growing procedure is triggered to adjust the template to the organ actual layout. Our current implementation controls the region growing method by means of a requirement of connectivity in the region, and also by keybord-introducing a range of allowable intensity pixel values. Note that the region growing may turn out to be a region shrinking procedure depending of the image data.

3. The procedure so far described applies to the current slice; however, it does not have to be repeated for every slice, but we exploit the fact that a great spatial redundancy exists in volumetric image data between two consecutive slices. This is implemented by projecting the segmentation result onto the two consecutive slices, and the triggering a new region growing procedure on the two neighbouring slices using this projection as the seed. The procedure is finished when the region eventually dissapears (i.e., no connected image pixels within the intensity bounds exists).

4. Part of the segmentation protocol is a region modification module; the user can delete regions or portions of regions at will, either in one particular section or throughout the volume. Also, new pieces of the images can be added to existing regions.

Although simple, this procedure gives enough interactivity so as to create graphical models out of 3D ecographies (the rendered images shown in the paper have been segmented with this tool by a non-expert operator). However, surfaces so created are prone to suffer degradations due to the lack of smoothing operations between slices. To alleviate this problem we have a module of research algorithms some of which have drawn interesting results [13. Also, active contours allow the user to apply less effort in the initial sketch of the regions to be segmented. However, the proper characteristics of the ultrasound data make 


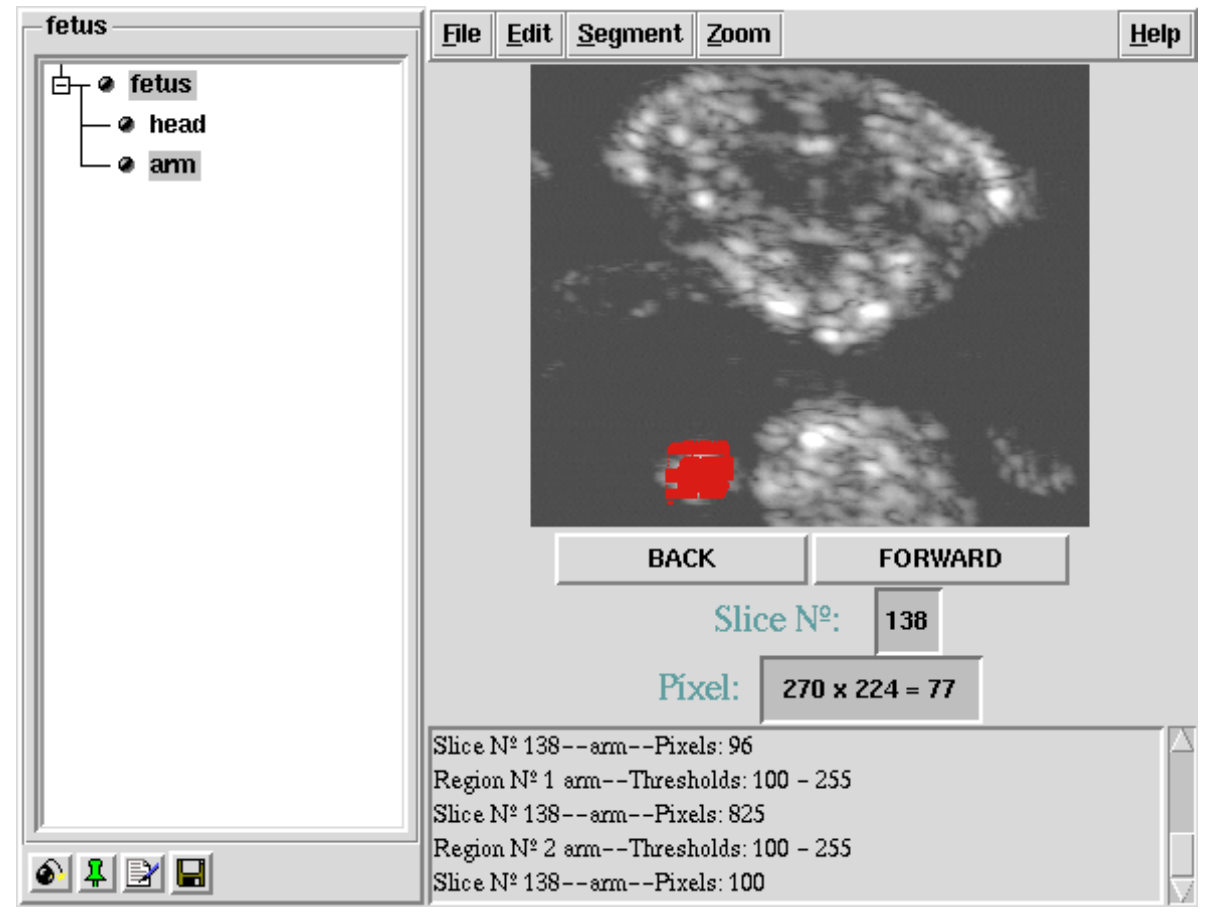

Fig. 2. Non-collaborative SMTool. The user can draw on the regions to be segmented, and the data structure is created and shown on the data tree structure on the left hand side.

energy function selection an issue[14]; we are currently porting this module into the diSNei platform.

Finally, the segmenter allows the user to create the hierarchical data structure we have mentioned before. It also creates the logic needed to generate graphical models out of the labelled data.

\subsection{Medical Image Visualization with diSNei}

Our environment diSNei incoporates advanced visualization facilities that have quite the same features as other reported browsers. However, the design has been oriented at maximizing the ergonomics and ease of use. Specifically:

- The gap between segmented (labelled) volume data and graphical model is filled in by means of an isosurface extraction module. Though the algorithm to calculate the isosurfaces is well-known [15] it is worth mentioning that this complexity is completely transparent to the end user, since the functionality is guided by the data structure that has been created in the segmentation phase. Therefore a few mouse-guided operations are the only thing to be done by the application end user. 


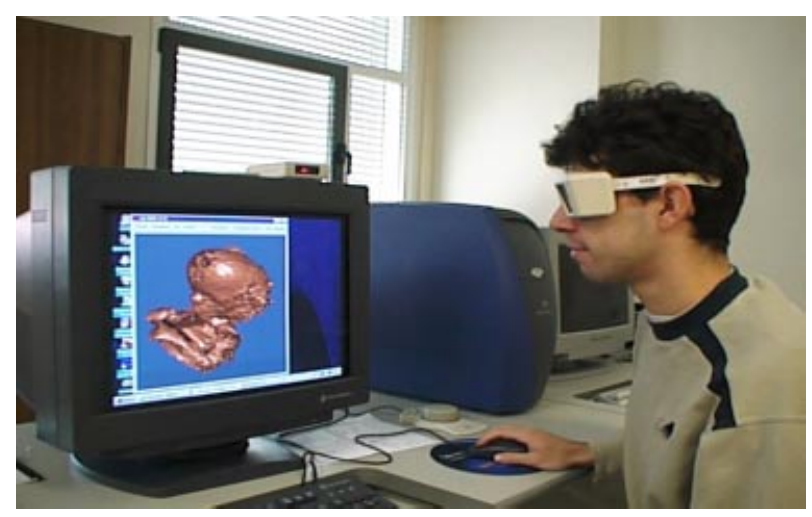

Fig. 3. The stereo-rendered fetus

- Once the graph model is created, the user selects which organs to represent, the colors to be used, and whether the representation should be done semitransparent or opaque. Once again, this is done by the menus on the left-hand side of the browser (see figure 1). Functional relations can be highlighted in the graphical model by proper selection of an entire tree branch.

- A number of predefined views can be selected from a menu. This increases speed in representation since no rotations are needed to achieve positions of interest.

- Navigation throughout the graphical model is a built-in feature in our application. The user can fly through the outer surface of the graphical model so as to see inner structures from inside.

- Our current version includes stereo rendering. By using stereo glasses the feeling of inmersion is dramatically improved at no further computational cost (in a Sillicon Graphics NT workstation). The glasses are also comfortable to wear and they only need a small controlling device (placed on top of the monitor in figure 3) so no further cables are needed.

- A plane can intersect the model with axial, sagittal and coronal planes (see figure $4 \mathrm{a}$ ) and a plane with an arbitrary position and orientation (figure 4b), The volume data are resliced according to the plane orientations and displayed. With this module, the end user can see at a glance where every slice in the raw data is actually located in $3 \mathrm{D}$ space.

- Data can be exported to other environments, specifically, a virtual reality modelling language (VRML) browser.

\subsection{CSCW with diSNei}

Human interaction is a very useful source of knowledge when it comes to solve a difficult problem. An example of this interaction are the so-called brainstorm meetings in which the interactors put in common ideas with the ultimate purpose 


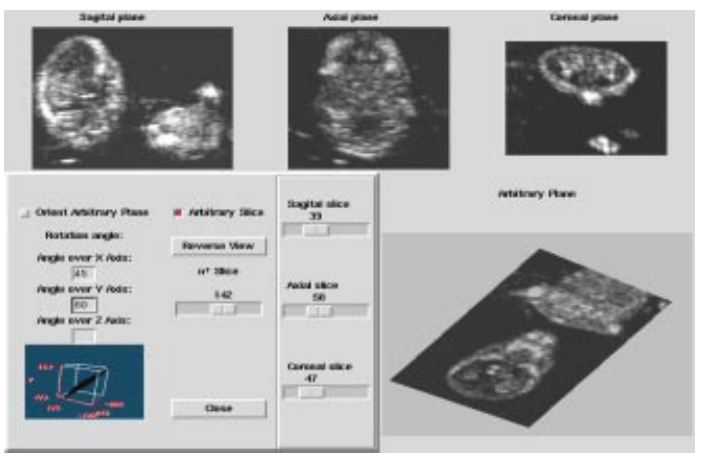

a)

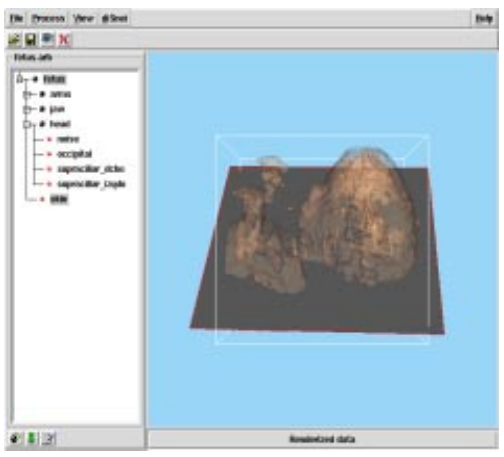

b)

Fig. 4. a) Sagittal, axial and coronal planes (top) together with an arbitrarily oriented plane (bottom). Volume data are properly interpolated to get this representation. b) Graphical model with the intersected plane.

to come up with an interesting overall idea created from contributions of the participants. Computer technology has made this collaboration possible by the so-called CSCW or, more generally, electronic groupware 16.

CSCW is a hot topic that is under use in many different scenarios. As far as the medical imaging field is concerned, several implementations have been reported during this decade. Most of them have to do with one or two dimensional signals 9 17 18 19, which are so far the ones typically used by physicians in their daily practice. However, we adhere to Thomas Berlage [10] in believing that graphical models of volume medical data create the common ground that is needed for useful interactions. This is specially true in the case of fetal echographies, since there is no scanning protocol widely accepted, but every echographist has his/her proper style. Once the volume data is acquired with a traditional free-hand two dimensional probe, image interpretation is not so straightforward. Indeed, unless some provision is made, the spatial data of the scanning procedure itself is lost (i.e., the absolute position and orientation of the ultrasonic probe), so it cannot be used as an additional piece of information for cooperation.

In diSNei we are aware of how important it could be for a physician to be assisted in some cases by a colleague, so we have created a collaborative environment in which at least two such physicians may collaborate to jointly reach a proper decision. Some of the foregoing ideas have been generalized in order to simultaneous and coordinatedly be carried out by more than one user. To be more specific, several users (see figure 51) can exchange information on the echographies both by text-based messages and also by free-hand drawing on the data; the arrows in the bottom of figure 5 5 ) give the possibility to scroll back and forth in the slices of the volume data. Collisions in synchronous actions are avoided by means of a token-grabbing procedure: the user who wants to perform any task with the shared data needs to grab the token. Once the token 


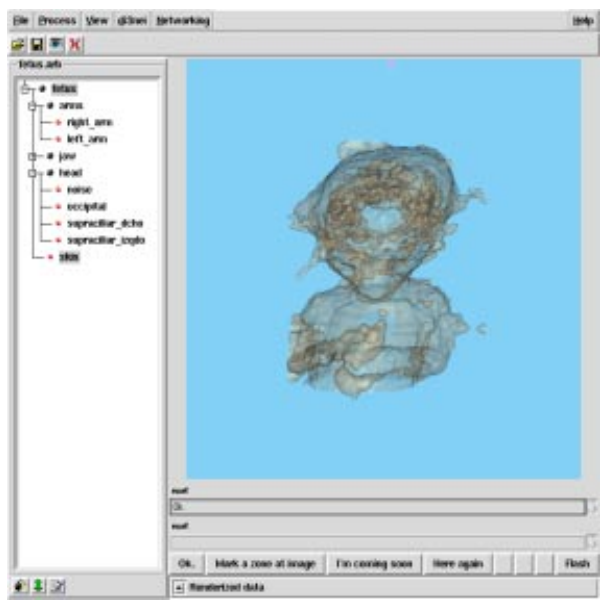

a)

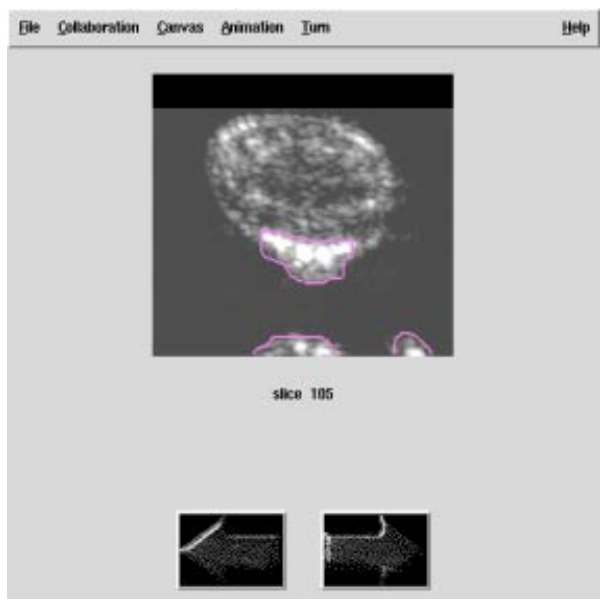

b)

Fig. 5. CSCW version of diSNei. a) Browser and talk channel b) CSCW-SMTool. The arrows allow any user to scroll forward or backward in the slices of the volume data.

is grabbed, the rest of the participants in the conference become listeners. The users can also manipulate the graphical model at will, with the same access protocol as before. The text-based information exchange is asynchronous, so any user can communicate with the rest of the participants at any time. This communication channel is the natural way to let the users agree a respectful token grabbing procedure.

Our platform uses the GroupKit package [20] which is a Tcl/Tk application programming interface (API) that allows programmers to create group activites fast and reliably. Most of the coordination is taken care of by the API; the programmer is therefore allowed to focus on the final application.

\section{Conclusions}

In this paper we describe a novel collaborative environment for volume data analysis and inmersive visualization; the package currently contains an easy-touse module for volume data segmentation and a very ergonomic stereo-rendering environment, which gives a high degree of inmersion in the graphical model. All the operations can be performed coordinatedly by several simultaneous users, regadless of their actual physical location. A steady communication channel is always available for physicians to exchange typed messages at any time.

We are currently working on different tiers to improve the system performance and capabilities. One effort is focused on developing compression algorithms for multiresolution data visualization; this module could be useful in the case that the application were to be used with a low-bandwith channel. Moreover, for clinical purposes, direct measures of organs and structures on 3D space 
seem mandatory. As far as the collaboration is concerned, the application is still in its infancy, but several ideas are currently under development. Some of them are the use of powerful database management tools for information retrieval and exchange, and also the implementation of a commond ground that includes the information of the actual scanning procedure. To this end, we are using magnetic position devices as reported in [1].

\section{Acknowledgements}

This paper has been partially supported by research grants TIC97-0772, and 1FD97-0881. The authors want to thank the students Miguel Ángel Toribios and José Carlos de la Fuente for their help in much of the programming of some collaborative aspects of diSNei and Marco Pinacho, Alberto Rivera and Eduardo Suárez for their help in the SMTool. Prof. Yannis Dimitriadis at the University of Valladolid should also be mentioned for the useful discussions with the authors and for all the on-going research on the CSCW in diSNei. Finally, the authors are indebted to Med. Dr. Arya Nabavi at University of Kiel (Germany) and Brigham \& Women's Hospital (USA) for many fruitful discussions on clinical aspects of this work and to prof. Yang at Saskatchewan University (Canada) for kindly sharing with us the ultrasound data we have used in our research.

\section{References}

1. Solus Project, http://svr-www.eng.cam.ac.uk/Research/Projects/Solus.

2. Invivo Project, http://www.igd.fhg.de/teleinvivo

3. Golland et al. AnatomyBrowser: A Framework for Integration of Medical Information. MICCAI98 (Proc of the First International Conference, MA, Cambridge, USA, Springer Lecture Notes in Computer Science, 1998, Vol. 1679, pp.720-731.

4. Brigham \& Women's Hospital and Harvard Medical School. Surgical Planning Lab, http://www.splweb.bwh.harvard.edu:8000

5. Gering et al. An Integrated Visualization System for Surgical Planning and Guidance Using Image Fusion and Interventional Imaging. In C.J. Taylor and A. Colchester (eds.): Medical Image Computing and Computer-Assisted Interventions, Lecture Notes in Computer Science, Vol. 1679. Springer-Verlag, Berlin Heidelberg, New York (1999) 809-819.

6. Mayo Clinic. ANALYZE software, http://www.mayo.edu/bin/analyze/\$ANALYZE_Main.html

7. Sensor. MEDx software, http://www.sensor.com/\$medx_info/medx_docs.html

8. MNI. MNI software, http://www.bic.mni.mcgill.ca/software

9. Y. Bouillon, F. Wendling, F. Bartolomei, Computer Supported Collaborative Work (CSCW) in Biomedical Signal Visualization and Processing, IEEE Trans. on Info. Tech. in Biomed. 3 (1999) 28-31.

10. T. Berlage, Augmented Reality Communication for Diagnostic Tasks in Cardiology, in Biomedical Signal Visualization and Processing, IEEE Trans. on Info. Tech. in Biomed. 2 (1998) 169-173.

11. W. Schroeder, K. Martin, B. Lorensen, The Visualization Toolkit: an objectoriented approach to 3D graphics, Prentice Hall Int., New Jersey (1998). 
12. B. B. Welch, Practical Programming in Tcl/Tk, Prentice-Hall Int., New Jersey (1997).

13. R. San José, A. Rivera, M. Pinacho, C. Alberola, J. Ruiz-Alzola, A Kalman filter technique applied to surface reconstruction and visualization from noisy volume data. In E. Kramer (eds.): Ultrasonic Imaging and Signal Processing, Proceedings of the SPIE, Vol. 3982, San Diego, California (2000) 396-407.

14. M. Martín, E. Rodríguez, D. Tejada, C. Alberola, J. Ruiz-Alzola, Energy Functions for the Segmentation of Ultrasound Volume Data using Active Rays, Proc. of the IEEE Int. Conf. On Acoustics, Speech and Signal Processing, ICASSP-2000, Istambul, Turkey (2000) 2274-2277.

15. W. E. Lorensen and H. E. Cline, Marching Cubes: a High Resolution 3D Surface Construction Algorithm, Comp. Graph. 21 (1987) 163-169.

16. C. A. Ellis, S. J. Gibbs, G. L. Rein, Groupware: Some experiences and issues, Comm. of the ACM, 34 (1991).

17. L. Kleinholz, M Ohly, Supporting Cooperative Medicine: The Bermed Project, IEEE Multimed. Mag. (1994) 44-53.

18. L. Makris, I. Kamilatos, E. V. Kopsacheilis, M. G. Strintzis, Teleworks: A CSCW Application for Remote Medical Diagnosis Support and Teleconsultation in IEEE Trans. Inform. Technol. in Biomed, 2 (1998) 62-73.

19. E. J. Gómez, F. del Pozo, E. J. Oritz, N. Malpica, H. Rahms, A Broadband Multimedia Collaborative System for Advanced Teleradiology and Medical Imaging Diagnosis in Biomedical Signal Visualization and Processing, IEEE Trans. on Info. Tech. in Biomed. 2 (1998) 146-155.

20. GroupLab of University of Calgary, http://www.cpsc.ucalgary.ca/projects/grouplab/groupkit/ 